\title{
Demographic predictors of resilience among nurses during the COVID-19 pandemic
}

\author{
Davood Afshari, Maryam Nourollahi-darabad* and Niloofar Chinisaz \\ Department of Occupational Health Engineering, School of Public Health, Ahvaz Jundishapur University of \\ Medical Sciences, Ahvaz, Iran
}

Received 10 September 2020

Accepted 7 November 2020

\begin{abstract}
.
BACKGROUND: Coronavirus disease 2019 (COVID-19) is mostly transmitted through respiratory droplets. One of the exposure methods pf this disease is through occupational exposures and, thereby, a large number of people are prone to catching this disease due to their occupations. Nurses during the COVID-19 pandemic are at the forefront of healthcare. There is no information about the level of resilience and the demographic and job factors predicting resilience in the critical conditions of this occupational group.

OBJECTIVE: The present study aims to determine the resilience score and its predictive demographic factors among the nurses working at the hospitals involved with COVID-19 in Ahvaz, Iran.

METHODS: 387 nurses from Ahvaz hospitals participated in this study. The Connor-Davidson Resilience Scale (CD-RISC) was used to assess resilience. Demographic information was also collected using a designed questionnaire. Since the present study was conducted during the COVID-19 pandemic, the questionnaires were sent online. Data were entered into software SPSS (version 23) and $T$-test, ANOVA and regression methods were used for data analysis.

RESULTS: The mean score of $61.18( \pm 14.8)$ was obtained for CD-RISC. The results of this study showed that age ( $r=0.610, P=0.003)$, work experience $(r=0.572, P=0.030)$, and level of education $(r=0.514, P=0.044)$ had a significant positive correlation with nurses' resilience score during the COVID-19 pandemic. Multiple regression analysis indicated that work experience and level of education were the predictors of nurses' resilience $\left(R^{2}=0.15\right)$.

CONCLUSIONS: The score of nurses' resilience was low. Based on the results, education and work experience were determined as the contributing factors for resilience. The findings can help to better understand effective and predictive demographic factors to achieve higher resilience in stressful situations.
\end{abstract}

Keywords: Demographic predictors, coronavirus disease 2019, nurses, resilience

\section{Introduction}

Coronavirus disease 2019 (COVID-19) is a new disease that was discovered in 2019 and had not been previously diagnosed in humans. The disease was

\footnotetext{
*Address for correspondence: Maryam Nourollahi-darabad (PhD), Department of Occupational Health Engineering, School of Public Health, Ahvaz Jundishapur University of Medical Sciences, P.O. Box: 61355-131, Ahvaz, Iran. Tel.: +98 613373 8269; Fax: +98 613373 8282; E-mail: maryam.nourollahi@gmail.com.
}

first reported in Wuhan, Hubei Province, China, in late December 2019. The common clinical symptoms of this disease include respiratory signs, fever, cough, shortness of breath, respiratory disorders, and indigestion. In more severe cases, this infection can cause pneumonia, severe acute respiratory syndrome, kidney failure, and even death [1].

A novel coronavirus (2019-nCoV), the cause of COVID-19, is mostly transmitted through respiratory droplets by patients. One of the contact methods is occupational exposures, which have made a large 
number of individuals prone to this disease due to their job. Accordingly, the Occupational Safety and Health Administration (OSHA) has divided job tasks into four risk exposure levels, namely very high, high, medium, and low risk [2].

Healthcare workers are placed in high and very high risk groups based on the type of tasks that they do. In this regard, nurses are at the forefront of controlling and treating this pandemic, which represents their commitment and compassion, but they have in fact risked their lives while performing their responsibilities. The incidence rates of COVID-19 among the medical staff in China and Italy have been reported to be $10 \%$ and $9 \%$, respectively [3]. The rapid spread of COVID-19 in different countries has imposed high pressure on nurses and led to the emergence of stress in nurses in such a way that this stress is likely to affect the degree of their resilience [4].

In Iran, the first definitive case of COVID-19 diagnosis was reported on February 19, 2020 [5]. With the passage of ten days from the first case of COVID19 death in Iran, the spread of this disease increased from 19 to 31 provinces, and, thereby, Iran became one of the global centers of COVID-19 [6]. The results of an epidemiological study conducted in one of the hospitals of Tehran have shown that the number of 12870 patients has referred to the emergency ward of the hospital from February 19, 2020 to April 15,2020 . It is worth noting that 2968 out of the 12870 patients have been hospitalized as cases diagnosed with COVID-19 [7]. The first study carried out in one of the southern provinces of Iran also demonstrated that 440 COVID-19 patients had been hospitalized up to March 19, 2020 [8]. The monitoring system in Iran identifies and reports new cases every day. Therefore, due to the high prevalence of COVID-19, the healthcare system has been strongly influenced and, as a result, a great deal of physical and psychological pressures have been imposed on the medical staff in caregiving to the COVID-19 patients. Excessive workload, the large number of patients, and the lack of equipment have all led to the imposition of much stress on nurses. In addition to the above-mentioned factors, the lack of knowledge about the disease and concerns about family and children are the issues that have brought about fear, anxiety and burnout in nurses [9-11]. The results of a study conducted on ICU nurses also showed that they are exposed to high stress, which in turn has led to fatigue, sleep disorder, nervousness, and frequent crying; in particular, young nurses have experienced more mental crises $[12,13]$. Considering the challenges faced in a pandemic, one of the factors that helps nurses show their best performance is their resilience or ability to maintain healthy and sustained psychological function despite exposure to severe stressors. Resilience is a multidimensional feature that helps a person successfully adapt him/herself to challenges and leads to the increased compatibility and enhanced protection against stressful situations. Resilience is learnable and, according to psychologists, factors such as individual and personality traits, family ties, and support systems reinforce it [14-16]. During the COVID-19 pandemic, nurses are at the forefront of healthcare, but no specific information is available about the level of resilience and the demographic factors predicting resilience in the critical conditions of this occupational group. Hence, the current study aims to: 1) determine the degree of resilience among the nurses working at the hospitals involved with COVID-19, 2) determine the relationship between reported resilience and demographic factors, and 3) determine the demographic factors predicting resilience among nurses at Ahvaz hospitals.

\section{Methods}

\subsection{Participants and sampling}

Nurses working at the hospitals in Ahvaz were invited to participate in this descriptive analytical study, when the confirmed and suspected cases of patients with COVID-19 were rapidly increasing and the coronavirus conditions were in the red alert state according to the official statistics of the Ministry of Health of Iran. The criteria for the inclusion of participants in this study were the work experience above one year and having no chronic physical or mental illness by self-report. The required data were collected using Connor-Davidson Resilience Scale and a questionnaire pertaining to demographic characteristics.

Since this study was conducted during the COVID19 pandemic, the questionnaires were submitted to the respondents in the online mode through https:// porsline.ir/ in order to reduce contacts and face-toface visits. Moreover, the consent form was sent to the participants online so that they could announce their agreement in taking part in this research. At the same time, the necessary explanations about the research objectives and how to answer the questions were also sent to the individuals online. For each person, only one response to the questionnaire was permitted. This study was approved by the Ethics 
Review Committee of the Ahvaz Jundishapur University of Medical Sciences (reference number IR.AJUMS.REC.1399.704).

\subsection{Measurements}

\subsubsection{Connor-Davidson Resilience Scale}

In this study, the 25-item Connor-Davidson Resilience Scale was used. This questionnaire can distinguish resilient from non-resilient people in clinical and non-clinical groups and can be used in research and clinical situations. In this questionnaire, five subscales, namely personal competence, trust in one's instinct and tolerance of negative affect, positive acceptance of change and safe relationships, control, and spiritual influences are assessed [14-17].

The Connor-Davidson Resilience Scale contains 25 items that are scored based on Likert scale from not true at all (0) to true nearly all of the time. Thus, the questionnaire score ranges from 0 to 100 where higher scores represent a greater degree of resilience. In fact, resilience is characterized by a CD-RISC score higher than 80. In this regard, Connor and Davidson have reported the Cronbach's alpha coefficient of the Resilience Scale to be 0.89. Moreover, the reliability coefficient of 0.87 has been reported for this scale through test retest method within a four-week interval [18]. The validity and reliability of this questionnaire were assessed in a study by Derakhshanrad et al. [19] and cronbach alpha coefficient was calculated to be 0.89 .

\subsubsection{Demographic information questionnaire}

The current demographic factors were selected based on the related literature and, then, a questionnaire with questions on age, gender, marital status, number of children, sports activity, work experience, education, hospital classification, field of study, type of employment, and organizational conditions was designed.

\subsection{Data analysis}

Analyses were performed using SPSS version 20 (IBM Co. LTD, Chicago, IL, USA). T-test, ANOVA, and post-hoc tests were run to compare the score of resilience among different demographic groups. Pearson correlation analysis was used to examine the correlation of resilience with demographic variables. Multiple linear regression analysis was used to examine the demographic variables predicting resilience. In all tests, the significance level was $0.05(P<0.05)$.

\section{Results}

\subsection{Demographic information}

Out of the 699 visits paid to the questionnaire, 387 questionnaires were filled out, which is representative of the return rate of $55 \%$. In the present study, approximately $60 \%$ of the participants were women and $40 \%$ were men. In terms of marital status, $48.5 \%$ of the participants in the study were single, while the remaining $46 \%$ were married. In addition, $39.5 \%$ had no children. With regard to sports activity, $6 \%$ of the participants used to exercise, but were not doing any exercises at that time due to the conditions caused by the COVID-19 pandemic; however, 52\% of them did not exercise at all. More details on the demographic factors under study are given in Table 2.

\subsection{Resilience}

The mean score of $61.18( \pm 14.8)$ was obtained for CD-RISC. The mean score of resilience and its subscales are presented in Table 1 . The maximum and minimum scores were 96 and 26, respectively. Besides, it was revealed that $12 \%$ of the study population were high resilience (CD-RISC score >80). Figure 1 shows the distribution of CD-RISC.

Table 2 shows the CD-RISC score based on the participants' demographic characteristics in the study. The female participants obtained significantly lower scores of CD-RISC than the male participants $(P<0.05)$. The results of statistical tests showed that there was a significant difference in CD-RISC scores among different age groups $(P<0.05)$. The results of the post-hoc comparison showed that this difference is linear in various age groups in such a way that the lowest CD-RISC score belonged to the age groups of smaller than 25 years, 25-30, 35-40, 30-35, 40-45, and above 45 years old, respectively $(P<0.05)$. There was a significant difference in CD-RISC score among groups with different work experiences $(P<0.05)$ in

Table 1

Mean and standard deviation $($ mean \pm SD) of resilience subscales $(\mathrm{N}=387)$

\begin{tabular}{lccc}
\hline CD-RISC subscale & Score range & Mean & SD \\
\hline Personal competence & $0-32$ & 19.34 & 5.52 \\
Trust in own intuition & $0-28$ & 15.2 & 4.36 \\
Accept change & $0-20$ & 13.22 & 2.87 \\
Control & $0-12$ & 6.88 & 2.56 \\
Spiritual influences & $0-8$ & 5.63 & 1.73 \\
Connor-Davidson & $0-100$ & 61.8 & 14.8 \\
$\quad$ Resilience Scale score & & & \\
\hline
\end{tabular}




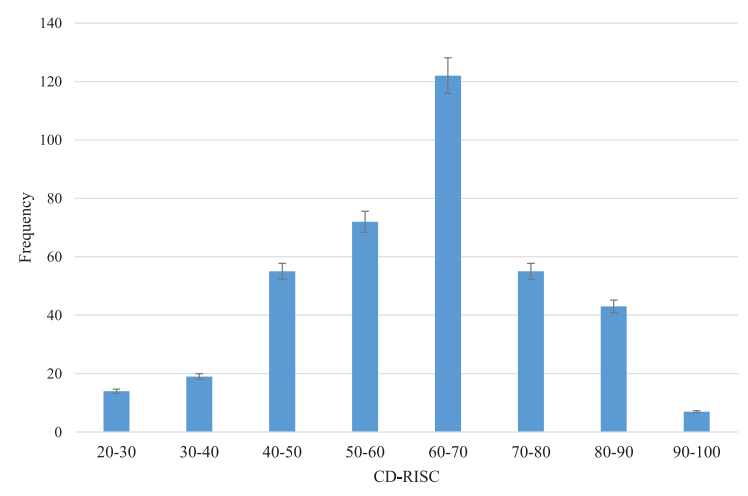

Fig. 1. Distribution of the Connor-Davidson Resilience Scale (CD-RISC) score $(\mathrm{N}=387)$.

Table 2

Demographic information and CD-RISC score (N:387)

\begin{tabular}{|c|c|c|c|}
\hline Category & $\mathrm{N}$ & $\begin{array}{c}\text { CD-RISC score } \\
(\text { Mean }( \pm \mathrm{SD}))\end{array}$ & $p$-value \\
\hline \multicolumn{4}{|l|}{ Sex } \\
\hline Female & 236 & $59.53(14.9)$ & \multirow[t]{2}{*}{$0.03^{*}$} \\
\hline Male & 151 & $62.36(14.46)$ & \\
\hline \multicolumn{4}{|l|}{ Age (years) } \\
\hline$\leq 25$ & 73 & $59.65(15.37)$ & \multirow[t]{6}{*}{$0.04^{* *}$} \\
\hline $25-30$ & 94 & $59.39(15.95)$ & \\
\hline $30-35$ & 80 & $60.34(14,5)$ & \\
\hline $35-40$ & 51 & $61,67(14,57)$ & \\
\hline $40-45$ & 46 & $64,21(12.91)$ & \\
\hline $45 \geq$ & 43 & $69.6(10)$ & \\
\hline \multicolumn{4}{|l|}{ Marital status } \\
\hline Married & 178 & $62,01(13.7)$ & \multirow[t]{3}{*}{$0.34^{* *}$} \\
\hline Single & 188 & $60,5(15.73)$ & \\
\hline Other & 21 & $60,08(14.8)$ & \\
\hline \multicolumn{4}{|l|}{ Parent status } \\
\hline Childless & 168 & $62.339(13 / 4)$ & \multirow[t]{2}{*}{$0.66^{*}$} \\
\hline With child & 219 & $60.36(14.1)$ & \\
\hline \multicolumn{4}{|l|}{ Education level } \\
\hline BA & 245 & $60.02(12.6)$ & \multirow[t]{3}{*}{$0.03^{* *}$} \\
\hline MA & 122 & $61.67(15.3)$ & \\
\hline Higher & 20 & $63.23(13.42)$ & \\
\hline \multicolumn{4}{|l|}{$\begin{array}{l}\text { Work experience } \\
\text { (years) }\end{array}$} \\
\hline $1-5$ & 147 & $53,29(15)$ & \multirow[t]{4}{*}{$0.01^{* *}$} \\
\hline $5-10$ & 102 & $60.12(15.26)$ & \\
\hline $10-15$ & 66 & $61.27(8.3)$ & \\
\hline $15>$ & 72 & $67.73(13.4)$ & \\
\hline \multicolumn{4}{|l|}{ Hospital level } \\
\hline Private & 118 & $61,91(14,1)$ & \multirow[t]{2}{*}{$0.11^{*}$} \\
\hline Governmental & 269 & $60,33(14,4)$ & \\
\hline \multicolumn{4}{|l|}{ Employment type } \\
\hline Permanent & 129 & $62.07(13.3)$ & \multirow[t]{2}{*}{$0.02^{*}$} \\
\hline $\begin{array}{r}\text { Contract and } \\
\text { temporary }\end{array}$ & 258 & $60.48(14.2)$ & \\
\hline \multicolumn{4}{|l|}{ Sport } \\
\hline Yes & 161 & $63.51(14.5)$ & \multirow[t]{3}{*}{$0.36^{* *}$} \\
\hline Not now & 25 & $62.13(16)$ & \\
\hline No & 201 & $58.74(12.9)$ & \\
\hline
\end{tabular}

such a way that the participants with 10 to 15,5 to 10 , and 1 to 5 years of work experience had the highest resilience, respectively. Moreover, a significant relationship was also observed at different levels of education in such a way that the increase of education level led to the higher CD-RISC score. It was also revealed that the score of resilience reported in nurses with child was lower, although the difference between the score of resilience reported among the study groups was not significant. On the other hand, there was no significant difference in resilience between nurses with different marital statuses. The results of this study also showed that there was no significant difference between the individuals who did not exercise and those who exercised in terms of the level of resilience. However, the reported level of resilience in nurses who did not exercise was lower than that in those who exercised.

\subsection{Regression model of resilience and demographic predictors}

Pearson correlation coefficient was used to determine the correlation between resilience and demographic variables. The results showed that there was a significant positive correlation between resilience and demographic variables of age $(r=0.610, p=$ $0.003)$, work experience $(r=0.572, p=0.030)$, and level of education $(r=0.514, p=0.044)$. In addition, multiple linear regression was used to determine the resilience regression model based on predictive demographic factors. The results of regression modeling revealed that education and work experience remained in the model. According to the coefficient of determination $\left(\mathrm{R}^{2}\right), 15 \%$ of the resilience could be explained by the current variables (Table 3 ).

\section{Discussion}

\subsection{Resilience}

The aim of this study was to determine the degree of resilience and the predictive demographic factors of resilience in nurses during the COVID-19

Table 3

Multiple regression of demographic predictors of resilience

\begin{tabular}{lcccc}
\hline Demographic variable & B & S.E & $\beta$ & P \\
\hline Constant & 26.89 & 6.34 & & \\
Work experience & 0.96 & 0.48 & 0.22 & 0.012 \\
Education & 0.74 & 0.56 & 0.31 & 0.043 \\
\hline
\end{tabular}

$\mathrm{R}=0.39 \mathrm{R}^{2}=0.15$. 
pandemic. The results of the present study showed that the mean score of nurses' resilience during the COVID-19 disaster equaled 61.8 ( \pm 14.8$)$, which was lower than the resilience reported by Huang et al. among the medical staff in Radiology Departments (65.76) [1]. In the current study, only $12 \%$ of the participating nurses had a resilience score higher than 80 , which is smaller than the value reported by Mealer et al. where $21 \%$ of ICU nurses obtained a resilience score 92 and were highly resilient. It is noteworthy that research has not been undertaken in pandemic conditions [13]. Therefore, it can be argued that the prevailing conditions arising from to the spread of COVID-19 disease has negatively affected the percentage of highly resilient individuals and, thereby, the average score of resilience has been lower during the COVID-19 pandemic. In fact, the rapid spread of this disease and the increasing growth of the number of patients with the disease have made this phenomenon a major challenge when it comes to the disease prevention and control. Accordingly, such issues have resulted in a decrease in resilience among the nurses at the forefront of the fight against the disease.

\subsection{Demographic factors}

In the current study, the relationship of demographic factors, including age, gender, work experience, level of education, marital status, family status in terms of having children, sports activity, hospital classification, and type of employment with resilience was investigated. On average, women were reported to have a significantly lower resilience than men during the COVID-19 pandemic disaster. The results of a study conducted by Dai et al. during the COVID19 pandemic in China have demonstrated that there is a significant difference between male and female medical staff in terms of the level of concern about the development of the disease among themselves or their family members. Indeed, the female staff had higher levels of concerns [20]. Similarly, such a significant difference was also observed between the male and female groups in the study carried out by Campbell-Sills et al. [21]. In another study conducted in Iran to assess the health conditions and job satisfaction among healthcare workers during the outbreak of COVID-19, it was identified that female staff experienced more sadness and depression [22].

The difference in the score of resilience was not statistically significant between groups with different marital statuses and parent statuses. However, the nurses without children had a higher level of resilience. In this regard, the results of a study conducted by Zhu et al. on the immediate psychological impact of the COVID-19 pandemic on medical staff also showed that living with family and worrying about the self and family about getting infected with the disease have been among the factors contributing to anxiety and stress in medical staff [23]. In the same way, the results of a study about the H1N1 pandemic done by Goulia et al. also showed that the level of anxiety in healthcare workers was relatively high and the greatest concern pertained to the infection of family and friends as well as to the health consequences of this disease [24]. In fact, the lack of psychological and social support and the absence of a sense of responsibility towards children and family are likely to play an important role in reducing nurses' resilience during the COVID-19 pandemic. The results of the present study represented the availability of a significant difference in terms of resilience between the groups with different levels of work experience, age, and education where an increase in each of the three factors would lead to the increased level of resilience. In fact, it can be pointed out that the increase of nurses' age, work experience, and education; and their exposure to stress as well as their personal abilities and skills to cope with critical and stressful situations develop. The development of such skills helps with the creation of various coping strategies, which can facilitate their adaptation and provide them with the possibility to act effectively and more resiliently in such situations. Gillespie BM et al. carried out a study on the resilience of operating room nurses and reported that their resilience increased with the increase of age, experience, and education of nurses [25]. In this line, Campbell-Sills et al. also argued that the resilience score rises with an increase in the level of education [21]. Another study conducted in Iran on mental health and job satisfaction of nurses in the face of COVID-19 showed that the older medical staff had better mental health and education level was a predictor of physical and mental health [22]. Although not conducted in the critical condition of the COVID-19 pandemic, another study maintained that an increase in the nurses' age and experience would lead to their enhanced skill in stress management at work [26-29]. Therefore, for increasing resilience in medical staff with a lower level of education and work experience, it is highly necessary to provide them with the pertinent trainings that can increase their knowledge and skills in COVID-19 disaster management and coping. 
In terms of the type of employment and hospital classification, nurses with permanent employment as well as nurses working in private hospitals had higher levels of resilience. Another study conducted on healthcare workers in Iran during the COVID-19 pandemic has shown that the employees in private institutions benefited from more desirable mental health [22]. Different conditions in terms of the number of patients and working hours may have led to this difference.

In terms of sports activity and getting habituated to sports activities, it can be claimed that doing exercise can be a protective factor against anxiety and reduce the psychological effect of catastrophic events [30]. In the present study, the participants who did not exercise had a lower resilience score.

The results of correlation analysis also showed that work experience, education level, and age had a significant correlation with resilience. In the proposed linear model, only work experience and level of education were recognized as the predictor variables of resilience. The proposed model could explain approximately $15 \%$ of the resilience variance in the COVID-19 pandemic, while $85 \%$ of the resilience variance in these conditions has not been determined. Thus, other effective factors in nurses' resilience in critical situations like psychosocial factors should be taken into consideration and assigned credit by managers.

\subsection{Limitation}

Our study examined only nurses. Future studies should compare the resilience and risk factors between all personnel involved with COVID-19 patients.

\section{Conclusion}

In most countries, COVID-19 is currently a major challenge for the healthcare system. Nurses are at the forefront treating patients and, as a result, are under high stress, which can affect their resilience. The results of the present study showed that the level of nurses' resilience was low. According to the study results, education and work experience were determined as the contributing factors for resilience. Based on the study findings, to achieve higher resilience in the stressful situation, resilience training programs and increasing knowledge about working at critical situation arising from unknown diseases are recommended.

\section{Acknowledgments}

This study was financially supported by Ahvaz Jundishapur University of Medical Sciences (grant no. U-99255). The authors would like to thank all participants for their cooperation.

\section{Conflict of interest}

The authors declare that there are no conflicts of interest.

\section{References}

[1] Huang L, Wang Y, Liu J, Ye P, Cheng B, Xu H, Qu H, Ning G. Factors Associated with Resilience Among Medical Staff in Radiology Departments During The Outbreak of 2019 Novel Coronavirus Disease (COVID-19): A CrossSectional Study. Med Sci Monit. 2020;26.

[2] COVID-19 - Control and Prevention /Healthcare Workers and Employers. Available from https://www.osha.gov/ SLTC/covid-19/healthcare-workers.html

[3] Istituto Superiore di Sanita' (ISS). Sorveglianza Integrata COVID-19 in Italia 2020 [updated 26 March 2020; cited 26 March 2020]. Available from: http://www. epicentro.iss.it/coronavirus/bollettino/Infografica_26marzo \%20ITA.pdf.

[4] Smith GD, Ng F, Li WH. COVID-19: Emerging compassion, courage and resilience in the face of misinformation and adversity. Journal of Clinical Nursing. 2020;29(910):1425.

[5] Iranian Ministry of Health and Medical Education. COVID-19 daily epidemiology journal. http://corona.beh dasht.gov.ir/files/site1/files/Factsheet-12.23-En.pdf Date: 13 March 2020. (Accessed 2020 March 24)

[6] Mounesan L, Eybpoosh S, Haghdoost A, Moradi G, Mostafavi E. Is reporting many cases of COVID-19 in Iran due to strength or weakness of Iran's health system? IJM. 2020;12(2):73.

[7] Nikpouraghdam M, Farahani AJ, Alishiri G, Heydari S, Ebrahimnia M, Samadinia H, Sepandi M, Jafari NJ, Izadi M, Qazvini A, Dorostkar R. Epidemiological characteristics of coronavirus disease 2019 (COVID-19) patients in IRAN: A single center study. Journal of Clinical Virology. 2020.

[8] Akbari A, Emami A, Javanmardi F, Pirbonyeh N, Fadakar N. Early epidemiological analysis of CoVID-19: first report from South of Iran. Res Sq. Epub ahead of print 2020.

[9] Sun N, Wei L, Shi S, Jiao D, Song R, Ma L, Wang H, Wang C, Wang Z, You Y, Liu S. A qualitative study on the psychological experience of caregivers of COVID-19 patients. American Journal of Infection Control. 2020;48(6):592-8.

[10] Bridges J. Covid-19: Supporting nurses' psychological and mental health. Journal of Clinical Nursing. 2020;29(1516):2742-50.

[11] Muller AE, Hafstad EV, Himmels JP, Smedslund G, Flottorp S, Stensland S, Stroobants S, Van de Velde S, Vist GE. The mental health impact of the covid-19 pandemic on healthcare workers, and interventions to help them: a rapid systematic review. medRxiv. 2020. 
[12] Shen X, Zou X, Zhong X, Yan J, Li L. Psychological stress of ICU nurses in the time of COVID-19. 2020;1-3.

[13] Mealer M, Jones J, Newman J, McFann KK, Rothbaum B, Moss M. The presence of resilience is associated with a healthier psychological profile in intensive care unit (ICU) nurses: results of a national survey. INT J NURS STUD. 2012;49(3):292-9.

[14] Matha D MD, PraDhan B, Rajesh SK. Correlates and predictors of resilience among baccalaureate nursing students. Journal of clinical and diagnostic research: JCDR. 2017;11(2):JC05.

[15] Grimes A, Sparke V, Rouen C, West C. Preparedness and resilience of student nurses in Northern Queensland Australia for disasters. International Journal of Disaster Risk Reduction. 2020:101585.

[16] Manzano García G, Ayala Calvo JC. Emotional exhaustion of nursing staff: influence of emotional annoyance and resilience. International Nursing Review. 2012;59(1): 101-7.

[17] Fu C, Leoutsakos JM, Underwood C. An examination of resilience cross-culturally in child and adolescent survivors of the 2008 China earthquake using the Connor-Davidson Resilience Scale (CD-RISC). Journal of Affective Disorders. 2014;155:149-53.

[18] Connor KM, Davidson JR. Development of a new resilience scale: The Connor-Davidson resilience scale (CD-RISC). DEPRESS ANXIETY. 2003;18(2):76-82.

[19] Derakhshanrad SA, Piven E, Rassafiani M, Hosseini SA, Mohammadi Shahboulaghi F. Standardization of connor-davidson resilience scale in iranian subjects with cerebrovascular accident. Journal of Rehabilitation Sciences \& Research. 2014;1(4):73-7.

[20] Dai Y, Hu G, Xiong H, Qiu H, Yuan X. Psychological impact of the coronavirus disease 2019 (COVID-19) outbreak on healthcare workers in China. MedRxiv. 2020.

[21] Campbell-Sills L, Forde DR, Stein MB. Demographic and childhood environmental predictors of resilience in a community sample. J Psychiatr Res. 2009;43(12):1007-12.
[22] Kakemam E, Raeissi P, Raoofi S, Soltani A, Sokhanvar M, Visentin DC, Cleary M. Occupational stress and associated risk factors among nurses: a cross-sectional study. Contemporary Nurse. 2019;55(2-3):237-49.

[23] Zhu Z, Xu S, Wang H, Liu Z, Wu J, Li G, Miao J, Zhang C, Yang Y, Sun W, Zhu S. COVID-19 in Wuhan: Immediate Psychological Impact on 5062 Health Workers. MedRxiv. 2020.

[24] Goulia P, Mantas C, Dimitroula D, Mantis D, Hyphantis T. General hospital staff worries, perceived sufficiency of information and associated psychological distress during the A/H1N1 influenza pandemic. BMC Infectious Diseases. 2010;10(1):322.

[25] Gillespie BM, Chaboyer W, Wallis M. The influence of personal characteristics on the resilience of operating room nurses: A predictor study. International Journal of Nursing Studies. 2009;46(7):968-76.

[26] De Gieter S, Hofmans J, Pepermans R. Revisiting the impact of job satisfaction and organizational commitment on nurse turnover intention: An individual differences analysis. International Journal of Nursing Studies. 2011;48(12):1562-9.

[27] Afshari D, Mohammadi A, Saki A, Movafaghpour M. Continuous monitoring of back postures using portable inclinometer among nursing assistants. Iran Occupational Health. 2014;11(3):30-9.

[28] Aliabadi SF, Ostadtaghizadeh A, Ardalan A, Eskandari M, Fatemi F, Mirjalili MR, Khazai B. Risk analysis of hospitals using GIS and HAZUS: A case study of Yazd County, Iran. International Journal of Disaster Risk Reduction. 2020;101552.

[29] Lee JH, Hwang J, Lee KS. Job satisfaction and job-related stress among nurses: The moderating effect of mindfulness. Work. 2019;62(1):87-95.

[30] Zhang SX, Liu J, Jahanshahi AA, Nawaser K, Yousefi A, Li J, Sun S. At the height of the storm: Healthcare staff's health conditions and job satisfaction and their associated predictors during the epidemic peak of COVID-19. Brain, Behavior, and Immunity. 2020;87:144-6. 